\title{
The Research of Improving the Particleboard Glue Dosing Process Based on TRIZ Analysis
}

\author{
Huiling Yu, Delin Fan, and Yizhuo Zhang \\ Northeast Forestry University, 150040, No.26, Hexing Road, Harbin, P.R. China \\ \{Huiling.Yu, Delin.Fan, Yizhuo.Zhang, Yh12016\}@163.com
}

\begin{abstract}
This research creates a design methodology by synthesizing the Theory of Inventive Problem Solving (TRIZ) and cascade control based on Smith predictor. The particleboard glue supplying and dosing system case study defines the problem and the solution using the methodology proposed in the paper. Status difference existing in the gluing dosing process of particleboard production usually causes gluing volume inaccurately. In order to solve the problem above, we applied the TRIZ technical contradiction and inventive principle to improve the key process of particleboard production. The improving method mapped inaccurate problem to TRIZ technical contradiction, the prior action proposed Smith predictor as the control algorithm in the glue dosing system. This research examines the usefulness of a TRIZ based problem-solving process designed to improve the problem-solving ability of users in addressing difficult or reoccurring problems and also testify TRIZ is practicality and validity. Several suggestions are presented on how to approach this problem.
\end{abstract}

Keywords: Theory of inventive problem solving (TRIZ), 40 Inventive Principles, Cascade Control, Supplying glue system.

\section{Introduction}

Competitiveness in manufacturing creates a market demand for generating new effective ideas in the shortest time with the least use of resources. Engineers are taught various techniques for solving routine problems encountered in everyday practice. Several methods for enhancement the problem solving process such as brainstorming [1], synectics have been used with some success. Osborn's brainstorming method is a group problem-solving technique. Brainstorming groups generally consist of 5 to 10 people who work on a specific problem. These methods are relatively simple and can be learned quickly. However, they have not found much application in engineering due to their intuitive, stochastic approach to problem solving. Axiomatic Design by N.P. Suh [2] is useful in assessing the quality of various designs, and has proven that successful paradigms for dealing with creative processes can be developed on a systematic basis. These axioms enable quick evaluation of a proposed design rather than providing an algorithm for finding a comprehensive solution.In Table 1 below these methods are compared. The approach, methods associated with each approach, and the person most responsible for the method are identified. 
Table 1. Techniques for Enhancing Problem-Solving Ability

\begin{tabular}{lll}
\hline Approach & Method & Author \\
Psychological & Synectics & Gordon \\
Innovative knowledge-based & Brainstorming & Osborn \\
Innovative knowledge-based & TRIZ & Altshuller \\
Utilization of knowledge & Quality Function Deployment & Akoa \\
\hline
\end{tabular}

Theory of Inventive Problem Solving (TRIZ in its Russian abbreviation) is an analytical approach to creative engineering. It has been developed in the former Soviet Union by Genrikh Altshuller, starting in the fifties. The main postulate of TRIZ is as follows: Evolution of engineering systems is not a random process, but is governed by certain objective laws. Altshuller formulated eight laws of evolution of engineering systems. These laws can be utilized for conscious engineering system development briefly described below. Altshuller analyzed about 400,000 invention descriptions from different fields of engineering. The most effective solutions were selected and examined to reveal the objective laws (trends) of evolution of engineering systems. The evaluation of the solution's effectiveness was based on the concept of engineering contradiction: A problem becomes a creative one when an attempt to improve system's parameters by conventional means leads to deterioration of other parameters generates an engineering contradiction.

TRIZ was born as a problem-solving tool based on a systemic view of the technological world. The primary idea behind TRIZ approach to solving problems is that information about a specific problem must be first generalized, a solution concept has to be generated, and then the concept should be specialized in terms of a feasible solution. Traditional problem-solving methods aim to find a specific solution to a specific problem often using brainstorming or trial and error processes. In contrast, TRIZ translates the specific problem into an abstract problem or model of the problem and then to an abstract solution or model of the solution. Figure 1 depicts this strategy.

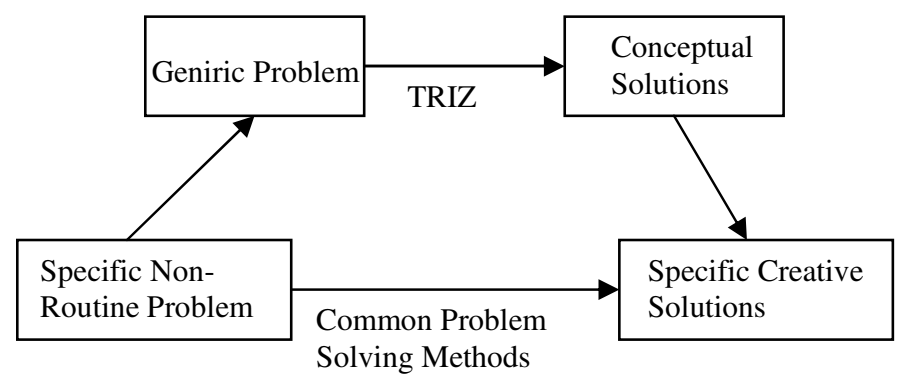

Fig. 1. TRIZ model for problem solving

TRIZ has been successfully applied in a number of industries to structure the innovation process. Mixing and supplying glue technology of particleboard is one of the key technologies on particleboard production line, although the investment of the entire section of all investment in whole line just accounted for $3 \%$ to $6 \%$, but it 
makes significant influence on whole line production. In the same process condition, excessive glue application will lead to high moisture content of particleboard, which can easily result in bubbling phenomenon and increased production cost at the same time, and formaldehyde emission will also be increased; if glue application is insufficient, the plasticity of particle will be small, the bending strength and plane tensile strength of particleboard will decrease with the reduction of glue application , which lead to the decline in the quality or substandard products.

As the glue flow is regulated in accordance with the shavings flow, it needs a period of time to regulate required flow glue by changing the speed of transmission plastic pump after flow meter received signal which causes a serious lag, only PID controller is hard to achieve the desired control effect. In addition, supplying glue effect not only depends on the controller's output, but also influenced by pressure changes inside pipeline and output pulsation caused by external interference in output and other factors, only controlling the frequency of the converter is difficult to achieve the stability and uniformity on supplying glue. Aiming to solve these problems, this paper presents a new method to identify and solve design problems in the conceptual stage synthesized the strengths of TRIZ's innovation and control theory's focus using a Smith Predictor.

\section{Application of the Theory of Inventive Problem Solving Tools}

Theory of Inventive Problem Solving (TRIZ in its Russian abbreviation) has been developed in the former Soviet Union by Genrich Altshuller, starting in the fifties. The main postulate of TRIZ is as follows: Evolution of engineering systems is not a random process, but is governed by certain objective laws. Altshuller analyzed about 400,000 invention descriptions from different fields of engineering.

TRIZ(the Theory of Inventive Problem Solving) had its inception in the mid-1940s with the efforts of Genrich Altshuller in understanding how engineered systems have evolved since the dawn of humankind. The TRIZ tools includes: the patterns of evolution of technological systems, ARIZ, technical contradictions and 40 inventive principles, the physical contradiction and 4 separation principles, substance-field analysis and 76 standard solutions and the technological effects and phenomena.

Altshuller defined a technical contradiction as the condition in which improvement of one system characteristic results in the degradation of another. He identified 39 system characteristics as being most often associated with technical contradictions.

\subsection{Gather Information about the Particleboard Glue Dosing System Problem}

\subsubsection{Describe the Particleboard Glue Dosing Problem in Non-technical Terms}

Gluing is the key process for the particleboard production, the adhesive which distributed evenly in the debris on the surface will have a direct impact on the quality of the finished product. We considered the factors impacting on the particleboard quality in the process of system analysis and got the sub-system and the super-system. We defined the Operational Zone $(\mathrm{OZ})$ and the Operational Time $(\mathrm{OT})$ then using the three-dimension analysis to get the Route Course(RC). To control the flow of the glue and the rotate speed of the pump according to the weight of the fiber. 


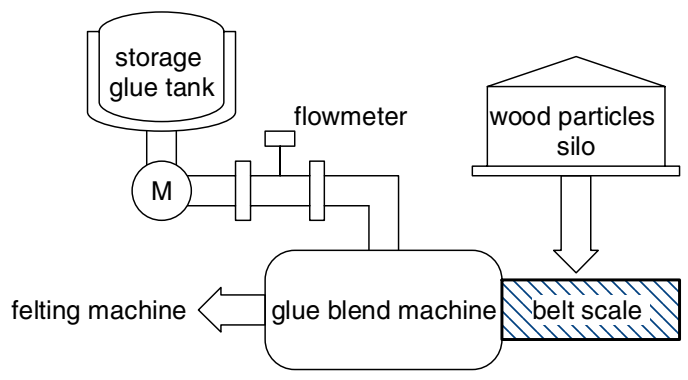

Fig. 2. Particleboard Supplying Glue System

\subsubsection{Define and Describe the Particleboard Glue Dosing System}

A system can be thought of as "a group of interacting, interrelated, and interdependent components that form a complex and united whole. Systems are almost always defined with respect to a specific purpose within a larger system. The system of supplying glue section in particleboard production line is composed of a glue storage tank, measuring devices, glue pumps, pipes and other components, divided into surface and core layer applying glue, with the same process and the symmetry structure. To solve the existed problem, the following supersystem in which the system resides were considered, such as air, the shape of flake, the pipelines and the density of the flake etc.

\subsection{Define the Way the System Functions}

When analyzing this case, the first step was to identify the useful functions/effects to achieve and/or the harmful ones to avoid in the case. The primary useful function of the glue dosing system is to measure flow accurately. Through the proportioned glue is stored in glue storage tank in accordance with the timing, supplying glue process is that shavings is sprayed by the corresponding glue mixture which is pumped from the two glue storage tank separately according to the weight of surface particle (thin stuff)and the weight of core layer and particle weight (coarse material), the particles is transported by Electronic belt scale, particle flow is measured precisely by rotary encoder, and glue content is measured by electromagnetic flow transmitter and load sensors , and then supplying glue steadily is realized though the method that using frequency converter to control the supplying glue pump.

\subsubsection{Define the System Boundaries and Environment}

TRIZ is a scientific methodology for defining, analyzing and formulating solutions to difficult problems usually of a technical nature. This research includes other systems nearby the supersystem: forming machine, hot press and sanding machine.

\subsubsection{Conditions Around the System and Its Supersystem Both Artificial and Natural}

Mixing and supplying glue system is a complex system with the characteristics that great time-delay, great inertia, multiple disturbance, nonlinear and time variation, and these characteristics make supplying control more difficult. 


\subsection{Refine the Definition of the Problem}

Since glue cost is high and the supplying glue control has a direct impact on hot pressing results of particleboard, the large time delay characteristic of supplying glue system affects product quality seriously. In addition, as the factors that electromagnetic interference, pressure variation inside and outside the pipe, mutations of shavings flow, failure shutdown etc exist in the control system during the supplying glue process, the traditional PID controller is difficult to achieve the required control quality of the system and manual intervention is often required.

\subsection{Define Available Resources}

Resources refer to the attributes of a system that can be used to improve the system including substances, fields, time, space, and environmental attributes. TRIZ looks for resources of innovation in the system itself not in the individuals or the organization surrounding it, so we can manage and guide the processes of value maximizing by problem solving. Any physical object with mass or that occupies space and that potentially can be used to improve a system calls substances. Includes Substance Resources: glue, flake, air etc, Space Resources: the length of the pipe, Time Resources: the time of flowing through the pipe, Information Resources: the flake's shape and density; Fields is defined as the energy needed for the interaction of substances within a system that can potentially be used to improve the system. Field resources: Chemical Field, electrical Field, mechanical Field.

\subsection{Consider Allowable Changes to the System}

The evaluation of the solutions' effectiveness was based on the concept of Engineer Contradiction: A problem becomes a creative one when an attempt to improve system's parameters by conventional means leads to deterioration of other parameters, i.e., generates an Engineering Contradiction. From the standpoint of TRIZ, solving a problem means overcoming this contradiction, or satisfying all conflicting requirements. 40 inventive principles (classes) of TRIZ are one of the less widely used innovation tools. Its have been the basis of the study for innovations in mechanism system.

Table 2. Contradiction matrix table

\begin{tabular}{|c|c|c|c|c|c|}
\hline \multirow{6}{*}{ 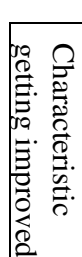 } & \multicolumn{5}{|c|}{ Characteristic that is getting worse } \\
\hline & \multirow{2}{*}{\multicolumn{2}{|c|}{ Engineering Parameters }} & 35 & 36 & 37 \\
\hline & & & $\begin{array}{l}\text { Adaptability } \\
\text { or Versatility }\end{array}$ & $\begin{array}{l}\text { Device } \\
\text { Complexity }\end{array}$ & $\begin{array}{ll}\text { Difficulty of } \\
\text { Detecting }\end{array}$ \\
\hline & 23 & Loss of Substance & & & \\
\hline & 24 & Loss of Information & & & \\
\hline & 25 & Waste of Time & & & \\
\hline F & 26 & Amount of Substance & & 3,13 & \\
\hline$\vec{n}$ & $\begin{array}{l}27 \\
28\end{array}$ & $\begin{array}{l}\text { Reliability } \\
\text { Accuracyof Measurement }\end{array}$ & & & \\
\hline
\end{tabular}


Altshuller defined 39 system characteristics as being the most often associated with technical contradictions. The contradiction table is a $39 \times 39$ matrix of all the characteristics on the rows and columns. To use it, first look up the character you wish to improve and go to its appropriate row. Next, locate the column of the characteristic that degrades as a result. Where the row and column intersect are numbers that correspond to the forty inventive principles.

The next step is to identify the standard and/or proposed contradictions in the case. In the supplying glue system, we found a main contradiction for the Macro-level and Micro-level that we wish to improve from the row 26 amount of substance, thus the undesired result — the characteristic that degrades as a result of improving the above feature, the column 36 device complexity. From the standpoint of TRIZ, solving a problem means overcoming this contradiction, or satisfying all conflicting requirements. Refer to the inventive principles whose numbers are listed in the intersecting cell. The inventive principles whose numbers are listed in the intersecting cell are: 3 Local Conditions principle; 13 Inversion principle; 27 Disposable Object principle; 10 Preliminary Action principle.

Among the four recommended principles, number 10 (preliminary action) provided feasible ideas to realize our IFR(Ideal Final Result). Problems that contain contradictions or conflicts can be solved one of two ways: either by making tradeoffs or by stating the objectives in the form of a contradiction and then solving the contradiction. Usually contradictions are resolved though trade-offs by finding some compromise between the two requirements. This results in a solution that does not fully satisfy either element. TRIZ, on the other hand, does not attempt to make tradeoffs between two requirements in features or functions. The foundation of Ideation TRIZ is the simple yet profound notion that the objective of design and innovation is to increase Ideality. Ideality of a system is defined by the following formula:

$$
\text { Ideality }=\text { Sum of the Benefits } / \text { (Sum of the Cost }+ \text { Sum of the Harm). }
$$

The sum of the benefits is defined as all of the useful effects and beneficial results from the functioning of a system.

The last step in the analysis is to identify the principles used in solving the problem and how they were used. Principle 10 preliminary action_-Arrange objects so they can go into action in a timely matter and from a convenient position, place each part of the object under conditions most favorable for its operation. Perform before it is needed.

Increasing system dynamism and controllability allows functions to be performed with greater flexibility or variety. We apply the cascade control system design based on predictor in advance. The Smith Predictor will be employed to the control system. Most of the principles suggested from the matrix match the concepts used in the actual solution of the problem.

The paper also considered solving the problem using a substance resource and field. Substance-Field analysis is a TRIZ analytical tool for building functional models for problems related to existing or new technological systems. Substances may be materials, tools, parts, people or environments. Fields may be mechanical, thermal, chemical, electrical or magnetic. 5 classes of 76 standard solutions are offered. According to sufield concept, model of the problem can be represented by $S_{1}$ (pump). To control $S_{1}$, a pair 
$\mathrm{F}_{-} \mathrm{S}_{2}$ should be introduced into the system. $\mathrm{S}_{2}$ is to transform $\mathrm{F}$ into mechanical field (force) which would act on $\mathrm{S}_{1}$. Due to ineffectiveness of mechanical $\mathrm{F}$, it was natural to suggest transition to microlevel which can be realized by use of control using a predictor.

\section{Supplying Glue System of Particleboard and Its Mathematical Model}

Organizations in order to survive and prosper have always needed to find solutions to difficult problems. However, current forces are impacting organizations and their management in ways unmatched in history. Forces such as accelerating rate of change, dramatic increasing competition, advancements in new technologies, and an increasingly complex information oriented world are just a few of the problematic issues facing organizations today. To succeed in such an environment organizations must find creative solutions to an array of organizational problems, in a multitude of organizational environments, with people of diverse backgrounds, knowledge bases and skill sets. This research examines the usefulness of a TRIZ based problem-solving process designed to improve the problem-solving ability of users in addressing difficult or reoccurring problems. For continuous mixing process, sizing control is to ensure that glue and particles are mixed into the glue blend machine by a certain percentage of the weight, which is one of the key processes to guarantee the stability of the particleboard's quality.

As is well known, the implementation of a control law for induction motors based on rotor flux control requires estimation of modulus and phase of the rotor flux vector in the stationary frame. In order to estimate rotor flux components in the desired frame, either open loop observers, such as current model and voltage models, or closed loop observers, such as full and reduced order observers, can be implemented.

In this paper neglecting the dynamics of the electrical parts in the linear motor X-Y table, its velocity model can be represented as first-order differential equation for each axis:

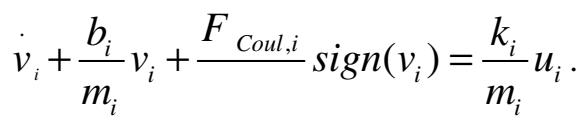

Where $v_{i}$ is velocity, $m_{i}$ is mass, $b_{i}$ is viscous damping, $k_{i}$ is input gain, $F_{C o u l, i}$ is coulomb friction and $i=x, y$, respectively.

Derived the simple and practical mathematical model of variable frequency pressure regulating supplying glue system in engineering, and when the voltage and frequency ratio of the power supply of squirrel-cage motors remains unchanged, its small deviation linear model is that:

$$
\begin{gathered}
J \dot{\omega}=-\left(D+p K_{0}\right) \omega+p K_{0} \omega_{1}-p m . \\
K_{0}=\frac{p}{r_{2}}\left(\frac{V_{10}}{\omega_{10}}\right)^{2} .
\end{gathered}
$$


Where $\omega_{1}, \omega$ are the deviations of Stator power frequency and rotor electric angular velocity separately; $V_{10}, \omega_{10}$, are the value on static work point of voltage and frequency of the Stator power supply; $p$ is role pair number; $r_{2}$ is the rotor resistance converted to Stator side; $\mathrm{D}$ is friction coefficient; $\mathrm{m}$ is the deviation of load torque.

For the pump load, its torque is proportional to the square of the rotational speed, and linearized by the small deviation, the relationship of the deviations become that:

$$
m=K_{m} \omega .
$$

Where $K_{m}$ is a constant. After formula (4) is entered in formula (3) and (2), it is got that:

$$
J \dot{\omega}=-\left(D+p K_{0}+K_{m}\right) \omega+p K_{0} \omega_{1} .
$$

Converted to transfer function is that:

$$
\frac{\omega(s)}{\omega_{1}(s)}=\frac{K_{d}}{1+T_{d} s} .
$$

The mathematical model of "pump-channel" part is a set of partial differential equations, to facilitate analysis, the glue flow response curve of this part can be got by test method, and then fitted with appropriate linear parts. The test indicates that the pump-pipeline part can be described with the following transfer function:

$$
\frac{h(s)}{\omega(s)}=\frac{K_{b}-K_{g} s}{1+T_{b} s} .
$$

\section{Cascade Control System Design Based on Smith Predictor}

The large lag characteristic is settled by Smith predictor control commendably, and dual-adjustment of converter frequency and motor speed realized at the same time, and the anti-interference ability and fault tolerance of the system improved too.

\subsection{Process Introduction on Supplying Glue System}

Single-loop control system is linked with $D(s)$, which can compensate the timedelay. This aspect of compensation is known as the predictor. Its transfer function: $G_{p}(s)\left(1-e^{-\tau s}\right), \tau$ is a constant of time-delay, $D(s)$ is a regulator's transfer function, $G_{p}(s) e^{-\tau s}$ is a transfer function of object. The compensation loop which is composed of Smith predictor and D (s) of is known as the time-delay compensation, the transfer function $D(s)$, that is:

$$
D^{\prime}(s)=\frac{D(s)}{1+D(s) G_{p}(s)\left(1-e^{-\tau s}\right)} .
$$


Closed loop transfer function is:

$$
\phi(s)=\frac{D^{\prime}(s) G_{p}(s) e^{-\tau s}}{1+D^{\prime}(s) G_{p}(s) e^{-\tau s}}=\frac{D(s) G_{p}(s)}{1+D(s) G_{p}(s)} e^{-\tau s} .
$$

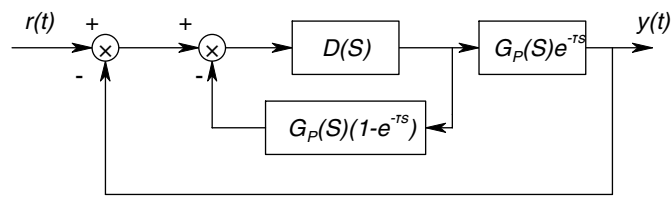

Fig. 3. Smith Predictor Principle Graph

Formula 9 indicates that the structure eliminates the pure delay part of the control system after compensation, as $e^{-\tau s}$ exists in the out of the closed-loop, the stability of the system can not be affected, only the control of the timeline goes on a time $\tau$, the transition process of control systems and other performance indicators are same to the $G_{p}(s)$, therefore, $D(s)$ can be designed in accordance with $G_{p}(s)$.

\subsection{Cascade Control System Based on Smith Predictor}

Cascade control system is developed on the basis of the single-loop control of the PID, which is a very common application of the control technology. Although the single-loop PID control mostly can complete control tasks as a variable is controlled, However, when a system variable is affect by several factors, or big time-delay exits in the controlled objects, load and interference change frequently or intensely, high controlling quality is required, or control task is rather special, a single-loop control is invalid. Cascade control system adds another PID single-loop control system as a subloop; the two single-loop control systems are combined by certain series the structure to achieve better control quality.

In this paper, aiming to achieve a static error control, the main loop choices PID controller, as shown figure 3, and the sub-loop choices PI controller, when the motor speed is changed by outside interference, the fast adjustment is implemented by PI control, so the interference $d(t)$ Motor inertia will not affect characteristics of the main loop.

\section{Simulation Analysis on Supplying Glue System}

According to the basic principles of cascade control, using Simulink programming, system is simulated in continuous mode, model parameters of speed regulation system are as follows: $K_{d}=1, T_{d}=60$, pumps and load model parameters: $K_{b}=1, K g=2.5$, $T_{b}=60$, the main loop PID controller parameters: $K_{p}=5, K_{i}=0.2, K_{d}=0.5$, sub-loop circuit PI controller parameters: $K_{p}=10, K_{i}=0.2$, time-delay constant $\tau=10$, and the sine function signal interference is added to sub-loop. The online model of cascade 
control is established and simulated, control output compares of system are shown in Figure 4. And Figure 5, we can see that cascade control has better quality control, especially in case of the system interference signals, cascade control has better Robustness and stability than the traditional PID controller.

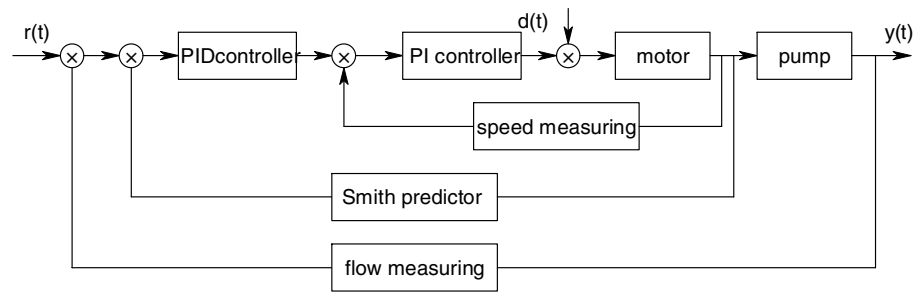

Fig. 4. Structure Graph of Cascade Control System

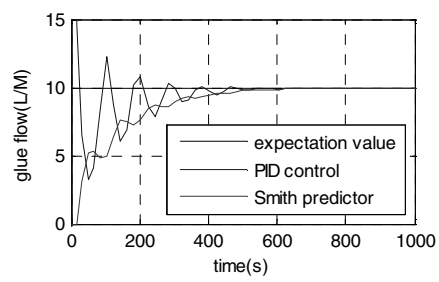

Fig. 5. Output Comparison With Noninterference

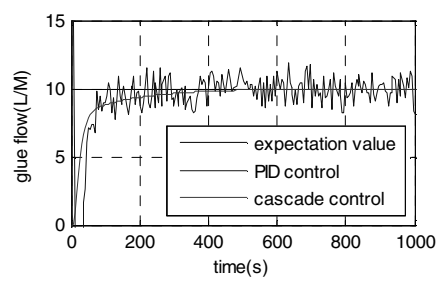

Fig. 6. Output Comparison With Interference

\section{Conclusion}

This paper introduced the theory of inventive problem solving(TRIZ) through applications in the field of particleboard glue dosing system. The Theory of Inventive Problem Solving (TRIZ) can be very helpful for developing non-obvious conceptually new solutions to complex engineering problems. The paper analyzed the supplying glue system problem, formulated the mini-problem and defined the conflicting elements, got the inventive principle 10 prior action to solve the contradict problem that using a Smith predictor control system in advance. TRIZ provides a powerful tool for solving conceptual design problems. Several examples of application of TRIZ for 
obtaining non-obvious solutions to real life engineering problems are described. The simulation results show that the prior action method using smith predictor has better robustness and stability, control quality are superior to the traditional PID control of the single-loop system, and also testify the TRIZ theory is of the practicality and validity. Even more important is the established fact that after using TRIZ techniques, even average engineers are becoming creative since they realize that the real life problems can be solved by a logical analysis supported by an algorithm and not necessarily by revelations.

\section{Acknowledgements}

This study is supported by the Basic National Project Foundation of China (no. 2007FY140300).

\section{References}

1. Osbern, A.: Applied Imagination. Scribner and Sons, New York (1963)

2. Suh, N.P.: Axiomatic Design as A Basis for Universal Design Theory. In: Universal Design Theory. Aachen Shaker Verlag (1998)

3. Altshuller, G.: Innovation Algorithm:TRIZ, systematic innovation and technical creativity Technical Innovation Center, 1999. Examples. Triz-journal (2001)

4. Mann, D., Winkless, B.: 40 Inventive (Food) Principles With Examples. Triz-journal (2001)

5. Savransky, S.D.: Engineering of Creativity: Introduction to TRIZ Methodology of Inventive Problem Solving. CRC Press, Boca Raton (2000)

6. Abd El-Razek, M.E., Basha, I.M.: Constructability improvement of bridges using Stepping formwork. J. Constr. Eng. Manage. 127(3), 206-213 (2001) 